\title{
Gravitational Model of the Three Elements Theory
}

\author{
Frederic Lassiaille \\ University of Nice Sophia-Antipolis, Valbonne, France \\ Email: lumimi2003@hotmail.com
}

Received March 8, 2012; revised March 25, 2012; accepted April 15, 2012

\begin{abstract}
The gravitational model of the three elements theory is an alternative theory to dark matter. It uses a modification of Newton's law in order to explain gravitational mysteries. The results of this model are explanations for the dark matter mysteries, and the Pioneer anomaly. The disparity of the gravitational constant measurements might also be explained. Concerning the Earth flyby anomalies, the theoretical order of magnitude is the same as the experimental one. A very small change of the perihelion advance of the planet orbits is calculated by this model. Meanwhile, this gravitational model is perfectly compatible with restricted relativity and general relativity, and is part of the three element theory, a unifying theory.
\end{abstract}

Keywords: Relativity; Gravitation; Newton

\section{Introduction}

This article addresses an issue yielded by general relativity, which is a determination of a global space's shape. This absolute space-time is the general relativity spacetime, and this space deformation in space-time must be in conformity with Newton's law at least for long distances. The adopted point of view is a Euclidean relativity. A Euclidean mathematical context is used, with 4 dimensions (three of space, $x, y, z$, and one of time: $c t$ ). This is used for restricted relativity. For general relativity, of course we apply the same and we extend it with a tensor, except that here locally it is a Euclidean metric used to represent space-time. In one word, a Riemannian tensor is used, in place of the usual pseudo-Riemannian Minkowskian tensor.

The physics principles within this mathematical framework will be exactly the principles of relativity. There exists, however, a difference between relativity and the approach of this document. Indeed, constancy of spacetime distance, in a global representation of space-time. Minkowski's representation is not used, and the Lorentz's invariant length is left off.

At first, the method is postulating that Lorentz's equations are simply a consequence of space-time deformations by energy. In other words we try to express the general relativity "deformation" principle, in the context of restricted relativity. Once this is done, physics inconsistencies are revealed. Of course a solution is searched. This will finally lead to postulate the existence of indivisible particles, from which matter is made of. Thus, the space-time determination is calculated, by means of rela- tivity energy equation. By construction, this determination is coherent with Lorentz's equations. This is the final determination of the shape of global space inside space-time. After this theoretical construction, this article will describe some results of this gravitational model:

- Galaxy speed profiles mystery,

- Galaxy velocity mystery,

- Pioneer anomaly,

- Saturn flyby by Pioneer 11 ,

- Earth flyby anomalies,

- Perihelion advance or precession of Mercury and Saturn,

- Disparity of gravitational constant measurements.

The detailed about this article can be found in [1-3].

Reference [1] was the first article about the gravitational model of the three elements theory. It describes the theoretical basis of the model. It has been published in the $17^{\text {th }}$ NPA proceedings, vixra database, and on the site of the three elements theory, since 2010. It has also been published on Amazon site. Reference [2] was only published on the site of the three elements theory. But figures where extracted from it for publishing in the proceedings below. Reference [3] was published on the site of the three elements theory, vixra database, and leads to a short version of it, which is in progress in the following proceedings: 2011 Dark matter symposium (poster), $18^{\text {th }}$ NPA proceedings, PIRT 2011, ECLA 2011, FFP12, and may be SMFNS2011, STARS2011, and the $8^{\text {th }}$ International Conference on Progress in Theoretical Physics. These "proceeding versions" are also reminding briefly the content of [1], and using some figures of [1]. 


\section{Retrieving Lorentz's Equations}

Lorentz's physical context is used. Let us remind it. There are two inertial frames, $R(O, x, y, z, c t)$ and $R^{\prime}\left(O^{\prime}\right.$, $\left.x^{\prime}, y^{\prime}, z^{\prime}, c t^{\prime}\right)$, in uniform rectilinear motions at the $v$ speed one compared to another, along $O x$ axis. The $O^{\prime}$ point is moving along $O x$ axis in the direction of $x$ increasing. Here, only $x$ dimension, $c t$, and $x^{\prime}, c t^{\prime}$, are important. At $t$ $=x=0$ there is also $t^{\prime}=x^{\prime}=0$.

In order to find Lorentz's equations within this physics framework, and since our representations are Euclidean, we are bound to suppose that $O x^{\prime}$ axis rocked with an $\alpha$ angle compared to $O x$ axis, such as $\sin (\alpha)=v / c$. See Figure 1. In the same way it is necessary to have $O^{\prime}$ coordinates equal to: $\left(x=v t\right.$, and $\left.c t=v^{2} t / c\right)$. Conversely under these conditions the reader will be able to calculate that Lorentz's equations are found.

On the basis of this observation, we are tempted to suppose a coherent physics postulate. This postulate is the following.

Postulate 1. Any particle with a non null mass $m$, moving with $v$ speed along $O x$ axis, $x$ increasing, compared to an inertial frame $R(O x y z c t)$, deforms spacetime around it with a rotation of the Ox-Oct plan around the $O y O z$ axis, with an $\alpha$ angle between $O x$ and $O x^{\prime}$, such as $\sin (\alpha)=v / c$. During the displacement of this particle from $O$ to $A(x=v t, c t)$, a vacuum appeared inside spacetime. The location of this vacuum is the $\left(O, O^{\prime}, H\right)$ triangle, such as: $O^{\prime}$ coordinates are $O^{\prime}\left(v t, v^{2} t / c\right), H$ coordinates are $H(v t, O)$.

In the borderline case of a photon, with $v=c$, the swing becomes maximum: $\alpha=\pi / 2$, and the vacuum is the $(O, A, H)$ triangle.

Figure 2 represents the effect of postulate 1 . A $P$ particle is moving at $v$ speed in the inertial reference frame $R$, parallel to $O x$ axis, and in the direction of $x$ increasing. At the $t$ instant, the particle is located coordinates $x$ and $c t$ in $R$ ( $A$ point). Hence $R^{\prime}$ is also moving uniformly along $O x$ axis. It is the same case as the one of Figure 1, except that here exists the $P$ particle on $A$ point. On Figure 2, the space line rocked with the $\alpha$ angle, locally in $A$ and $O^{\prime}$.

On the other hand, far from $A$ point this line of space is

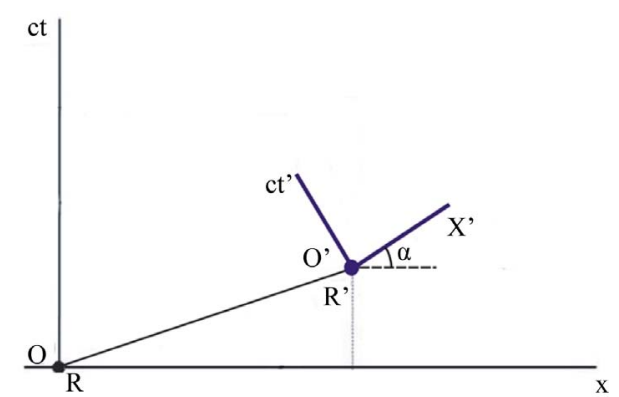

Figure 1. Lorentz transformation euclidean representation.

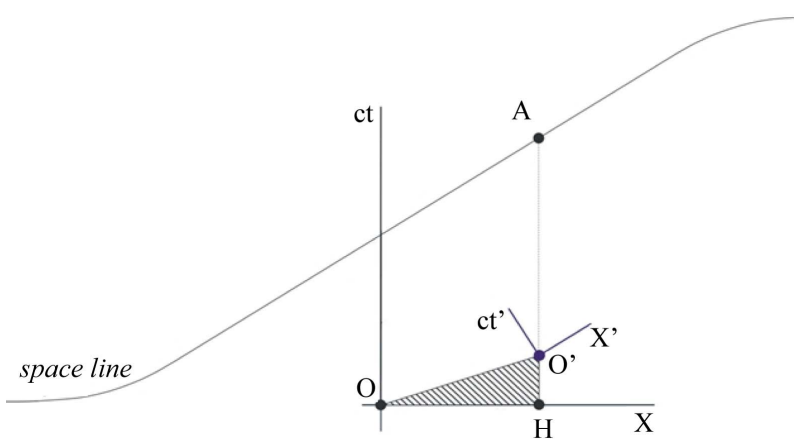

Figure 2. Postulate 1.

parallel to $O x$ axis. This is indeed the only realistic possibility! It is difficult to imagine the movement of a particle deforming the entire universe this way along $O x$ axis.

With this postulate, Lorentz transformation now expresses a local deformation of space-time, caused by the energy of the moving particle (postulate 1 above). This respect of Lorentz's equations is only local to the particle. It can be noticed that those restrictions explain the Sagnac effect.

\section{Luminous Points}

However at this stage a problem of coherence arises since a particle is constituted of smaller particles. Indeed, how to ensure that space-time deformation generated by the movement of a big particle, composed by a heap of smaller particles, can rise from the deformations of these smaller particles? To ensure this coherence a solution consists in supposing that matter is made up of a restricted group of very small "indivisible" particles. This is postulate 2 below.

Postulate 2. Each particle consists of a certain number of smaller particles, called the "luminous points". These "luminous points" are moving constantly at the c speed, inside the first particle, and with respect to any inertial frame of reference.

These small particles are conceived in such a way that they can explain space-time deformations generated by any other composed particle. For this explanation a simple operation must calculate the final deformation generated by the large particle, from the small particles it is made of. Thus defined, this operation must allow, by construction, calculation of the shape of absolute curves of space, starting from the positions and energies of these "small indivisible particles". At the same time, this operation must be, of course, compatible with postulate 1 and Lorentz transformation.

From this postulate 2 it is possible to determine in a single way any deformation generated by any particle. For that, we apply postulate 1 to these "luminous point" particles. For these luminous points the $\alpha$ angle is equal 
to its limit value $\pi / 2$. The shape of space is thus at any moment the result of successive combinations of these small deformations caused by all these "luminous points". What remains to be specified is the way of combining those various deformations. This will be specified by the postulate 3 which follows. After that, it will possible to check that the $\alpha$ angle calculated from postulates 2 and 3 is well given by the formula of postulate 1 .

For that let us return to Lorentz transformation. A first mathematical observation is essential. The energy conservation equation of restricted relativity is found by quantifying the luminous point trajectory lengths, inside a given $P$ particle. It is what we will see.

It will be supposed that this $P$ particle is modelled as consisting of only one luminous point. Consequently the obtained model is the one described by Figure 3. It can be checked that the reasoning remains valid in the general case of a particle made up of several luminous points.

When the $P$ particle moves from $O$ point (on Figure 3) to $A$ point, along $O A$ segment, the contained luminous point follows a trajectory having a $V$ shape, that is: 1) First stage: displacement at the speed $+c$ along $O x$, (milked in fat on Figure 3); 2) Second stage: displacement at the speed $-c$ along $O x$ (milked in fat on Figure $3)$. For the first stage, $L_{1}$ is the displacement length, and $L_{2}$, (positive), is the displacement length of the second stage. If $x$ is the position of the $A$ point, we can write: $x=$ $v t=L_{1}-L_{2}$. This $x$ position is also the space coordinate of $P$ in $R$ at this time $t$.

Hence:

$$
\begin{gathered}
c t=L_{1}+L_{2}, \quad v t=L_{1}-L_{2} \\
\frac{\left(L_{1}+L_{2}\right)^{2}}{2}=\frac{\left(L_{1}-L_{2}\right)^{2}}{2}+2 L_{1} L_{2}
\end{gathered}
$$

This last equation is the Pythagore equation using surfaces. It is also nothing more than the relativistic equation of energy:

$$
E^{2}=E_{c}^{2}+E_{m}^{2}
$$

with $E=m c^{2} / \sqrt{1-v^{2} / c^{2}}, E_{c}=m v c / \sqrt{1-v^{2} / c^{2}}$, and $E_{m}=m c^{2}$. To obtain the equivalent equation for the energy densities, each term of this equation is divided by the value $\left(L_{1}+L_{2}\right)^{2} / 2$ which is the value of the total energy of the particle:

$$
1=\frac{\left(L_{1}-L_{2}\right)^{2}}{\left(L_{1}+L_{2}\right)^{2}}+\operatorname{oper}\left(L_{1}, L_{2}\right)^{2}
$$

with

$$
\operatorname{oper}\left(L_{1}, L_{2}\right)=\frac{2 \sqrt{L_{1} L_{2}}}{L_{1}+L_{2}}
$$

The introduced operator is the relationship between the algebraic average and the arithmetic mean. It is equal to

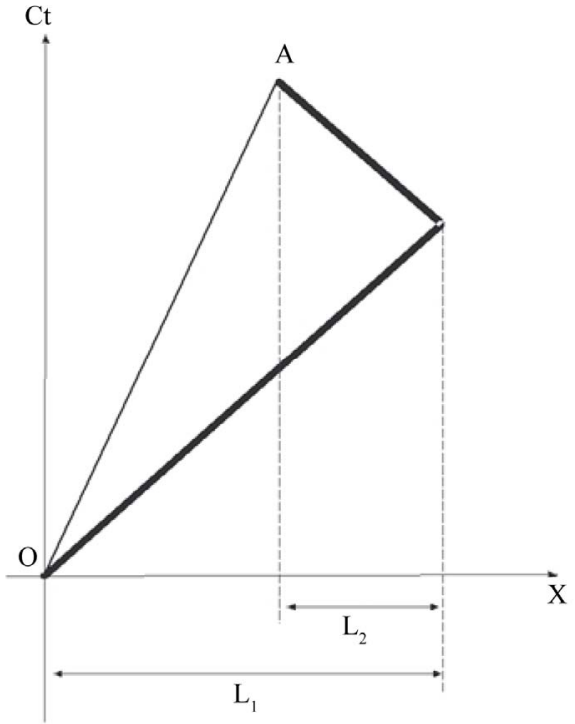

Figure 3. Luminous point trajectory in a moving particle.

the relativistic coefficient $\sqrt{1-v^{2} / c^{2}}$.

Indeed, from Equation (1): $2 L_{1} / c t=1+v / c$,

$2 L_{2} / c t=1-v / c$, and $1-v^{2} / c^{2}=4 L_{1} L_{2} /\left(L_{1}+L_{2}\right)^{2}$. Let us remind that Equation (4) is also written: $1=\sin ^{2}(\alpha)+$ $\cos ^{2}(\alpha)$ where $\alpha$ is the angle of the space-time swing of postulate 1 .

Finally, this last study of Lorentz transformation led us to define an operator. It will be used to postulate, finally, the mode of determination of general relativity absolute space-time. By construction, this determination will be compatible with restricted relativity.

\section{Relativistic Operator}

This is done by the following postulate. It generalizes the previous observation done about relativity energy equation.

Postulate 3. Space shape in space-time is given at any point by the ratio of the infinitesimal space lengths, $d s$ along space line, and $d x$ its length projected on $O x$ axis. This ratio is equal at any point to the relativistic operator applied to the two following values:

$L_{1}$ : sum of the heights of vacuum of space-time deformations propagated in Ox direction, $x$ increasing,

$L_{2}$ : sum of the heights of vacuum of space-time deformations propagated in Ox direction, $x$ decreasing.

That is to say: $\mathrm{d} x / \mathrm{d} s=2 \sqrt{L_{1} L_{2}} /\left(L_{1}+L_{2}\right)$ where $L_{1}$ and $L_{2}$ are the 2 above-mentioned sums.

This operator is neither linear nor associative. But this doesn't matter since it is calculated once, at any spacetime point. Its value remains the same, when calculated in different inertial frames. (This can be either deduced or directly calculated).

It is written above that $L_{1}$ and $L_{2}$ are the sums of 
the heights of vacuum of the "propagated deformations". It is necessary to describe how these "space-time deformations" are propagated. The mechanism is very similar to waves propagated by the movement of a boat over a water surface. Let us consider Figure 1. The initial deformation relates to the $O x$-Oct plan. The propagations of this deformations in space-time are carried out on remaining space dimensions, i.e. $\mathrm{Oy}$ and $\mathrm{Oz}$, more generally on any $O r$ direction, half-line based on $O$ and contained in the $\mathrm{Oy}-\mathrm{Oz}$ plan. The form of these propagated deformations is each time exactly the same as the one of the initial deformation. The initial deformation was done on $O x$-Oct plan (space-time swing represented Figure 1). Now the propagated deformation is the same but it relates to the $(O x+O r)-O c t$ plan in place of $O x-O c t$ plan. The height of this propagated deformation attenuates progressively as $r$ increases. The attenuation law, $g$, will be given further. At each moment, the "luminous point" thus emits this deformation. Therefore, like in the case of the boat, the finally overall propagated deformation is the envelope of all these propagated deformations. (In the case of a boat this envelope has a $V$ shape which is the final shape of these waves over the water surface). Here this envelope is a cone whose axis is $O x$ axis.

Let us study the overall result of all the propagated deformations which are received in the same $M$ point at the same moment. The postulate 3 above expresses the length ratio along $O x$ axis only. But at one $M$ point there are numerous such directions coming to. Hence it is necessary to calculate the relativistic operator of postulate 3 for each space direction. The final deformation is then obtained. The only question is: "What is the combination rule for the deformations of all these directions in order to obtain the result?" This result will be the resulting space-time deformation at $M$ point. It will be thus necessary to generalize the above operator with a second more generic operator, which will take into account each space directions. The result given by this second generalized operator must be the famous final space-time deformation at $M$ point. It will be probably useful to use a mathematical base like the quaternions for that. In this article this complexity will not be seen because fortunately not necessary.

We thus found relativity starting from postulates 1,2 , and 3. The $\alpha$ angle of the postulate 1 rotation is calculated, by applying postulates 2 and 3. Overall, we obtained a way for calculating space shape inside spacetime. We can now study Newton's law.

\section{Modification of Newton's Law}

The studied case is a particle of mass $M$ isolated in a space filled uniformly with a constant energy density. The particle coordinates are $x=y=z=0$, which are those of the $O$ point in our usual inertial frame $R$ of re- ference. The studied case being invariant by any rotation of center $O$, only the $O x$ axis with $x>0$, and the axis of times $O c t$, are important.

How does evolve the local slope $\tan (\alpha)$ of space, along $O x$ axis? The postulate 3 above is applied. Let us consider a space-time $P$ point to which comes at least one deformation from a luminous point pertaining to the $M$ mass. We suppose $P x$-coordinate positive strict that is $x$ $>0$. The $M$ mass particle propagates on $P$ point the following deformations, with propagation directions given:

$$
\begin{array}{ll}
L_{1 m}=g(x) & x \text { increasing } \\
L_{2 m}=0 & x \text { decreasing }
\end{array}
$$

For Equation (6), $g$ is the attenuation function given further. There is no deformation propagated in the direction of $x$ decreasing, coming from $M$, because $x>0$. The surrounding universe with constant energy density propagates on $P$ point the following deformations, with propagation directions given:

$$
\begin{array}{cc}
L_{1 u}=L_{u} & x \text { increasing } \\
L_{2 u}=L_{u} & x \text { decreasing }
\end{array}
$$

Therefore, the $L_{1}$ and $L_{2}$ sums are the following.

$$
\begin{gathered}
L_{1}=L_{1 u}+L_{1 m}=L_{u}+g(x) \\
L_{2}=L_{2 u}+L_{2 m}=L_{u} \\
\frac{\mathrm{d} x}{\mathrm{~d} s}=\operatorname{oper}\left(L_{u}+g(x), L_{u}\right)
\end{gathered}
$$

The last equation is the application of postulate 3. After calculations:

$$
\cos (\alpha)=\frac{\mathrm{d} x}{\mathrm{~d} s} \cong 1-\frac{g(x)^{2}}{8 L_{u}^{2}}
$$

In addition let's apply the formula of the expression of a force, to an $m$ mass moving. The traditional relativistic equation is the following one:

$$
F=\frac{m v \frac{\mathrm{d} v}{\mathrm{~d} x}}{\left(1-\frac{v^{2}}{c^{2}}\right)^{3 / 2}}
$$

This is a very classical relativity result. Now let us take the case of a particle with a negligible mass at rest. It is with this particular case that is applied the principle of general relativity: the trajectory of this particle will follow a space-time geodesic. Moreover, if the particle is located at rest infinitively far at $t=0$, then we have, for any $x, v=c \tan (\alpha)$, where $\alpha$ is the slope angle of the required curve $c t=h(x)$. This curve is the searched space curve. From where:

$$
F=m c^{2} \frac{\mathrm{d} \tan (\alpha)}{\mathrm{d} x} \frac{\tan (\alpha)}{\left(1-\tan ^{2}(\alpha)\right)^{3 / 2}}
$$


For $x$ large, this must be equal to $F=-m M G / x^{2}$, which is Newton's equation. Hence, after calculation, we have, for $x$ large:

$$
g(x)=L_{u} \sqrt{\frac{8 R}{x}}
$$

With $R=M G / c^{2}$. Now it will be postulated that this Equation (16) is correct not only for long distances but for any values of $x$. It will be supposed also that space is no longer filled uniformly with a constant energy density. Let us write $f$ as being the normalized space-time height of the propagated deformations which are coming from the closed surrounding matter. For example, in the case of a galaxy, this $f$ contribution will comes from the galaxy's stars. It will be used also $s=1+f$, where 1 stands here for the normalized contribution coming from the universe. With this notation, the $L_{1}$ and $L_{2}$ sums becomes the following.

$$
\begin{aligned}
& L_{1}=L_{u}\left(1+f+\sqrt{\frac{8 R}{x}}\right) \\
& L_{2}=L_{u}(1+f)
\end{aligned}
$$

After calculations, Equation (15) becomes the following.

$$
F=-\frac{m M G^{\prime}}{x^{2}} \frac{\left(s+\sqrt{\frac{2 R^{\prime}}{x}}\right)\left(s+2 x \frac{\mathrm{d} s}{\mathrm{~d} x}\right)}{\sqrt{s^{2}+s \sqrt{\frac{8 R^{\prime}}{x}}}\left(s^{2}+s \sqrt{\frac{8 R^{\prime}}{x}}-\frac{2 R^{\prime}}{x}\right)^{3 / 2}}
$$

In this equation, $G^{\prime}$ stands for the value of $G$ valid for long distances and in extra-galactic locations, and $R^{\prime}$ is equal to $M G^{\prime} / c^{2}$. It must be noticed that, if $f=0$, it still remains a correction of Newton's law expressed by Equation (19). But this modification is only noticed for $x$ close to the Schwarzschild radius $\left(2 R^{\prime}\right)$.

In this document, if $f=0$ then this Newton's law modification will be called the "first modification of Newton's law". If $f \neq 0$, it will be called the "second modification of Newton's law”.

\section{The Pioneer Anomaly}

This modification of Newton's law has been using a fitting of Newton's law for long distances. But for studying the Pioneer anomaly, this fitting must not be done for long distances. It must be done for a distance from the sun where the heliocentric gravitational constant is known to be perfect. This value is around the sun to Saturn distance. This can be seen on the measured curve of the Pioneer anomaly, in [4]. With this supposition the calculations yields a theoretical anomaly value equal to $A_{t}=7.25 \times 10^{-10} \mathrm{~m} / \mathrm{s}^{2}$, in place of the measured value
$A_{m}=8.74 \times 10^{-10} \mathrm{~m} / \mathrm{s}^{2}$. But the shape of the theoretical curve is not perfect. Figure 4 shows this theoretical curve. It has been plotted using Equation (19) and the "first modification of Newton's law".

There is also a negative predicted anomaly for the probe's trajectory between the sun and Saturn ( $x$-coordinate lesser than $10 \mathrm{AU}$ ). This work is in progress, and noticeably exact comparison with ephemerides must be done.

In order to retrieve the perfect curve, it must be supposed that $f$ is not null nor constant but varies ("second modification of Newton's law”). The Kuiper belt will be taken into account. The Kuiper belt is a belt of asteroids located beyond the location of Saturn, along the ecliptic plane. Now the result, on Figure 5, is very encouraging. On this theoretical curve, the maximum value is exactly $A_{m}=8.74 \times 10^{-10} \mathrm{~m} / \mathrm{s}^{2}$, the measured value. But this theoretical curve has been obtained with fitted values for the Kuiper belt space-time deformations contributions. On the contrary, the curve of Figure 4 was calculated without any fitting.

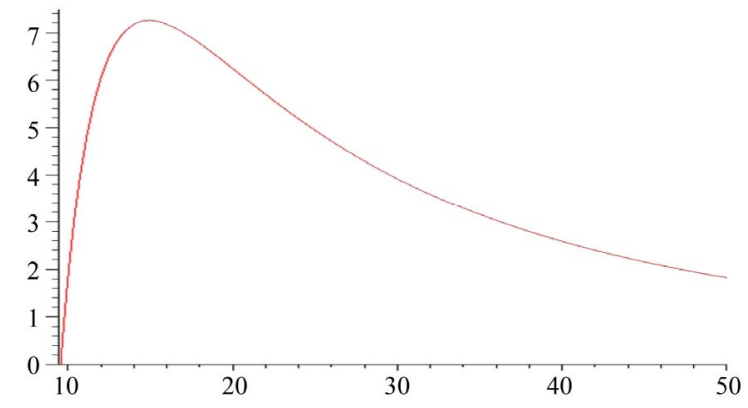

Figure 4. Theoretical curve of the Pioneer anomaly, using "first modification of Newton's law". $X$-coordinate is the distance from the sun, in $A U, y$-coordinate is the added acceleration toward the sun, in $10^{-10} \mathrm{~m} / \mathrm{s}^{2}$. This theoretical curve must be compared with the experimental one located in [4].

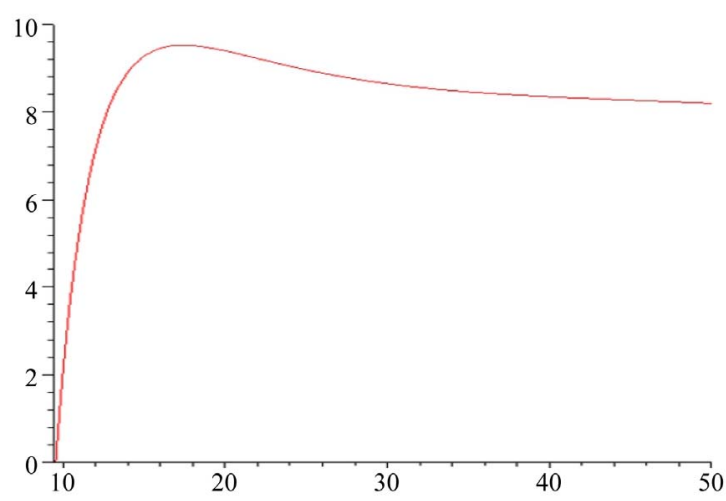

Figure 5. Theoretical curve of the Pioneer anomaly, using the Kuiper belt, and the "second modification of Newton's law" for calculation. $X$-coordinate is the distance from the sun, in $A U, y$-coordinate is the added acceleration toward the sun, in $10^{-10} \mathrm{~m} / \mathrm{s}^{2}$. 


\section{The Pioneer 11 Flyby of Saturn}

When applying this gravitational model to the case of Pioneer 11 trajectory, an added anomaly is found. It is an acceleration anomaly during the flyby of Saturn. The model is calculating an anomalous decrease before the Saturn encounter, and an anomalous increase after the encounter. This is shown on Figure 6. An important parameter has been fitted. It is $x_{0 s}$, the distance at which the Saturn gravitational constant, $G M_{s}$, is perfect $\left(M_{s}\right.$ stands for Saturn mass). Indeed, in this gravitational model, this distance is always of great importance: the distance between the attracted masses, where Newton's law is perfect.

The values of Figure 6 are very strong, as compared to the Pioneer anomaly values, but the distances range of this anomaly is very short. This range is roughly [9.550 $\mathrm{AU}$, 9.555 $A U$ ]. It has been checked that the global Pioneer anomaly curve is still correct for this Pioneer 11 travel, after taking into account this Saturn flyby anomaly. In fact, this global curve shape depends strongly on the $x_{0 s}$ value.

\section{Speed Profile Mystery}

In order to obtain the explanation of the stars speed in a galaxy it is necessary to take into account these star masses. For this, a $P$ point is supposed located in the middle of these stars i.e. inside the studied galaxy. It will be supposed that the deformation propagated by these stars and received in $P$ is only coming from the surrounding stars closed to $P$. This supposition is confirmed later on, outside of this article.

It will be supposed that this matter density in a galaxy evolves following a $1 / x^{2}$ law. ( $x$ is the distance from the galactic center). As a consequence, the $f$ contribution of Equation (19) is equal to $r / x$, where $r$ is the ray from which the gravitational effect of the surrounding stars is noticed. With those suppositions and notations, Equation (19) becomes:

$$
F \simeq-m M G \frac{x-r}{(x+r)^{3}}
$$

Now, of course $M$ stands for the mass of the galactic center. It is noticed that the gravitational force can be null, and even negative for $x<r$. As usual the tangential speed of the stars is calculated using the classical equation $v=\sqrt{F x / m}$.

This Equation (20) yields the curve located on the bottom of Figure 7. It has been used: $r=1 \mathrm{kpc}$. This value has been adjusted in order to obtain the best possible curve.

The variation of the speed of the stars in a galaxy is theoretically explained. Hence we retrieve the global

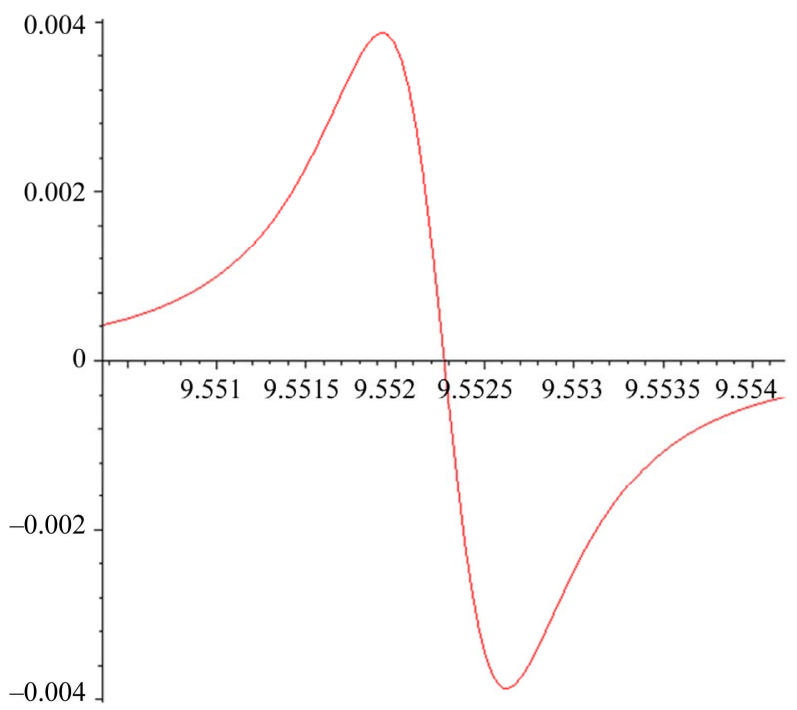

Figure 6. Theoretical curve of the Pioneer 11 anomaly, in the vicinity of Saturn. $X$-coordinate is the distance from the sun, in $A U$, and $y$-coordinate is the anomalous acceleration toward the sun, in $\mathrm{m} / \mathrm{s}^{2}$. The represented anomaly is the sum of the Pioneer anomaly and the Saturn flyby anomaly.

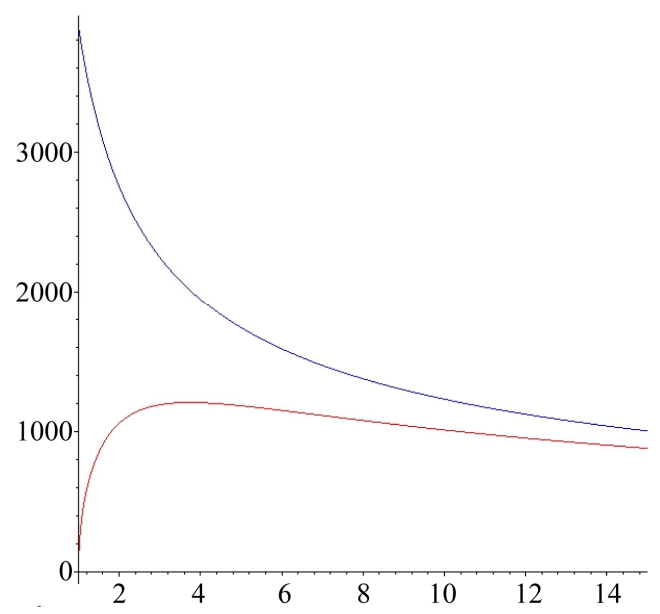

Figure 7. Theoretical speed profile for the stars in a galaxy, calculated using a $1 / x^{2}$ matter density curve. $X$-coordinate represents, in $k p c$, the distance between the star and the galactic center. The unit of the ordinate, $y$, is $\mathrm{km} / \mathrm{s}$. The curve located on the bottom represents the speed resulting from the new model. The curve located on the top represents the speed resulting from traditional Newton's law.

shape of a typical galaxy speed profile.

More practically, a program calculating the NGC 7541 speed profile yields the curve of Figure 8. This curve has been computed using the matter density profile available in [5]. The corresponding measured speed profile of NGC 7541 is also found in [5]. The shape of this measured curve is the same as the theoretical curve of Figure 8. But there is an issue of sign for the theoretical speed profiles of those real galaxies. Each time, locally, the 


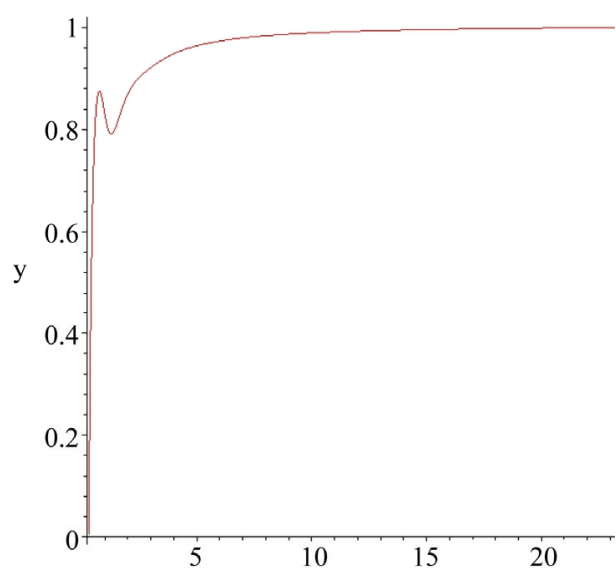

Figure 8. Theoretical speed profile of the NGC 7541 galaxy. $X$-coordinate is in $k p c$, and $y$ ordinate is a relative speed. The sign of the gravitational force has been changed, and the speed values has been raised with a fitted constant value, before normalization.

shape of the curve is not retrieved, but another one, which is exactly the same as the measured one, except that it is reversed along the $y$-coordinate. Hence, using an opposite sign for the gravitational force equation, the measured curve shape is retrieved. This is true for each of the five real galaxies which has been calculated: NGC 3310, NGC 1068, NGC 157, NGC 7541, and NGC 7331. This is calculated in [2]. A plausible explanation of this error is an occultation mechanism during the propagation of the space-time deformations coming from the galaxy luminous points. In this mechanism, the galaxy dust is acting like a fog, occulting the space-time deformation propagations coming from the matter located beyond it. This mechanism has been confirmed by calculations, but still remains to be explained fully.

For some galaxies, it is possible to calculate the exact values of the theoretical speed, for the maximums of the speed profiles. For NGC 3310, the measured value in [6] for the first maximum is around $175 \mathrm{~km} / \mathrm{s}$. The corresponding theoretical value is in the range $[20,700] \mathrm{km} / \mathrm{s}$. For NGC 1068, the measured value in [6] is $210 \mathrm{~km} / \mathrm{s}$, the theoretical value is $260 \mathrm{~km} / \mathrm{s}$. The precision of those theoretical calculations has not been evaluated. But those calculations are not fitted.

\section{Galaxy Speed Mystery}

Let us study the mystery of the velocities of the galaxies. From the above equations we have, after calculations:

$$
G=\frac{c^{4}}{\left(\sum_{p} \sqrt{\frac{8 e_{p}}{x_{p}}}\right)^{2}}
$$

This sum is done for each luminous point, $p$, along a half-cone. This half-cone is centered on the location in which we want to calculate $G . e_{p}$ is the energy of each luminous point. $x_{p}$ is the distance of each luminous point from the location in which we calculate $G$. Let us write:

$$
S_{i}=\sum_{p \text { inside }} \sqrt{\frac{8 e_{p}}{x_{p}}}, \quad S_{o}=\sum_{p \text { outside }} \sqrt{\frac{8 e_{p}}{x_{p}}}
$$

$S_{i}$ is for the $p$ luminous points inside the Milky Way. $S_{o}$ is for luminous points outside the Milky Way. Therefore:

$$
G=\frac{c^{4}}{\left(S_{i}+S_{o}\right)^{2}}, \quad G^{\prime}=\frac{c^{4}}{S_{o}^{2}}
$$

It has been written $G$ as the gravitational constant valid inside the Milky Way, and $G^{\prime}$ when located outside any galaxy. Therefore this modeling of relativity explains qualitatively the "dark matter" mystery for the velocities of the galaxies. Also, this explanation is the same for the mystery of light beam deviation in the vicinity of a galaxy.

\section{PPN Parameters}

The PPN parameters (Parameterized Post-Newtonian formalism) are exactly the same as for relativity. Indeed, the only differences between relativity and the gravitational model of the three elements theory are the following.

- Lorentz equations are true only between inertial reference frames which get "energy attached locally". "Energy attached locally" to a reference frame $(O, c t, x$, $y, z$ ) means that there is a particle or a group of particles whom inertial point is constantly equal to $O$.

- Newton's law is not used, like in relativity, but retrieved. There is a slight difference between Newton's law and this corrected Newton's law.

- There exists nonlinearity in the superposition law for gravity. This work is in progress.

Each relativistic equation remains also the same, except Einstein's equation because it is calculated using Newton's law. In the gravitational model of the three elements theory, Einstein's equation is only an approximation.

\section{Earth Flyby Anomalies}

The issue of the flyby anomalies is explained in [7]. When applying the "first modification of Newton's law", the order of magnitude is well retrieved for these anomalies. Table 1 shows the theoretical added velocities, which are roughly of the same order of magnitude as the measured ones.

However, those calculations are much too simpler. The 
Table 1. Comparison between the real and the theoretical Earth flyby anomalies for 6 probes. The values represents added perigee velocities. Units in $\mathrm{mm} / \mathrm{s}$.

\begin{tabular}{ccc}
\hline Probe & Measured anomaly & Theoretical Anomaly \\
\hline Galilleo I & 2.56 & 2.53 \\
Galilleo II & -2 & -11 \\
NEAR & 7.21 & -6.6 \\
Cassini & -1.7 & 4.5 \\
Rosetta I & 0.67 & 22 \\
Messenger & 0.008 & 29 \\
\hline
\end{tabular}

calculations must be done at least in the Schwarzschild's metric of the sun, using the motion of the Earth, the exact trajectory of the probes, and the contributions of the surrounding matter (asteroids, planets, etc.). Moreover, the "second modification of Newton's law must be used also, as the Pioneer anomaly analysis shows. Noticeably, the location of the ecliptic plane as compared to the exact probes trajectories, has an important impact on the final result. Indeed, the Kuiper belt is located on this plane, and the Kuiper belt influence has been proven in the Pioneer anomaly analysis above. This remark might explain the experimental verification of the importance of the location of the equator plane as compared to the probes trajectories. Indeed, the equator and the ecliptic planes are not far away from each other.

\section{Perihelion Advance}

When applying the "first modification of Newton's law" to the perihelion advance of Mercury and Saturn, the results of Table 2 are retrieved. The theoretical values are very close from the general relativity values. They do not explain the anomaly of the precession of the Saturn perihelion explained in [8], which is of -0.006 arc-second by century.

But, since the calculated values are very close to the GR values, it results that the three elements theory model seems to be compatible here with gravitation experimental measurements. Moreover, as well as for Earth flyby anomalies, the "second modification of Newton's law" must be conducted, in order to check if the Saturn anomaly of [8] is theoretically explained.

\section{Measurements of $G$}

The issue is well defined. For nearly three centuries, $G$ has been measured, without getting roughly a better precision than $0.7 \%$. The reason of this poor precision is the fact that many measurements values are contradicting each others, taking into account their confident interval. The details of this issue is, for example, well described in [9]. The "first modification of Newton's law" is not able to explain this issue. Indeed, the order of magnitude of the theoretical
Table 2. Theoretical modification of the perihelion advance or precession of Mercury and Saturn. On the left are the general relativity results calculated with a computer program. On the right are the added values from these general relativity values. Units are arc-second by century.

\begin{tabular}{ccc}
\hline Planet & GR value & 3elt value \\
\hline Mercury & 42.7848 & GR value -0.0014 \\
Saturn & 1.66291 & GR value +0.00056 \\
\hline
\end{tabular}

error is far below the experimental one.

But the "second modification of Newton's law" retrieves the same order of magnitude as the measured one.

Let's compare first with data coming from [10]. A prediction of the model of this document is that the $G$ value is depending of the surrounding matter distribution. This is not a new idea, it has been suggested in [11]. Moreover, it has been proven by experimental data in [10], that the value of $G$ depends of the orientation. This behavior is also a consequence of this theoretical idea. In [10], the amplitude of the variation of the $G$ value is more than $0.054 \%$ as compared to its absolute value. The prediction of the gravitational model of the three elements theory for this value is between $1 \%$ and $0.013 \%$ depending of galaxy matter distribution. Therefore, the order of magnitude of this model prediction is compatible with experimental data.

Now let's try another estimation, not taking in account the presence of the stars, like in [10], but the presence of mountains around the apparatus during the measurement of $G$. The ratio below is the relative difference between two values of $G, G_{1 m}$, and $G_{2 m} . G_{1 m}$ is the measured value of $G$ in [12], and $G_{2 m}$ is the measured value in [13].

$$
\frac{G_{1 m}-G_{2 m}}{G_{1 m}}=6.5 \times 10^{-3}
$$

Those two official measurements of $G$ have been chosen in order to get the greatest possible ratio above.

Let us assume that those two experiments have been done in completely different places. And the important difference between them is the distribution of matter in the surrounding neighbourhood.

- Experiment $\{1\}$, for example, is done at the very top of a hill on the floor of a desert, and this floor is completely plane outside of the hill on which we are located.

- Experiment $\{2\}$, is done in the middle of a valley, which is surrounded by mountains.

The interesting thing is that the measured value of $G$ will be completely different between those two cases even if exactly the same experiment apparatus is used and the same measurement procedure is applied. Indeed, the presence of the surrounding mountains in the second measurement has an important effect on the final measured 
value. After calculations, the corresponding theoretical ratio of $G$ is estimated by the following formula.

$$
\frac{G_{1}-G_{2}}{G_{1}} \simeq 2 \frac{r_{s}}{r_{g}} \sqrt{\frac{\rho_{s}}{\rho_{g}}}
$$

$r_{s}$ stands for the maximum distance between the surrounding mountains, and the location of experiment $\{2\}$. It will be used $r_{s}=4 \mathrm{~km}$.

$r_{g}$ stands for the greatest distance between a galactic object and the location of the experiments. It will be used the same value as for the Pioneer anomaly calculation: $r_{g}=140 \mathrm{pc}$. This value is based on the occultation mechanism solving the sign issue which has been noticed above for the speed profiles. In other words, galactic objects located beyond this distance will not be noticed. Their space-time deformation will vanish, during propagation, before arrival, because of the occultation mechanism. This $r_{g}$ value has been fitted in a coherent manner by the model when comparing its predictions to experimental data.

$\rho_{s}$ is the mean matter density of the surrounding mountains of experiment $\{2\}$. It will be used $\rho_{s}=1.7 \mathrm{~g} / \mathrm{cm}^{3}$, which is weaker than granite density $\rho_{\text {granite }}=2.7 \mathrm{~g} / \mathrm{cm}^{3}$.

$\rho_{g}$ is the matter density of the galaxy. We will use the value $\rho_{g}=0.709 \times 10^{-20} \mathrm{~kg} / \mathrm{m}^{3}$, which is the matter density in the galaxy, near the solar system.

With those numerical values, the final result is the following.

$$
\frac{G_{1}-G_{2}}{G_{1}} \simeq 0.9 \times 10^{-3}
$$

This theoretical value is not far from the measured one, of Equation (24). This proves that the order of magnitude of the measured difference can be explained by our correction of Newton's Law.

As an intermediate conclusion, the gravitational model of this study might explain the disparity between the measurements of $G$. This theoretical value of $G$ is depending on the distribution of matter in the surrounding neighborhood (buildings, hills, mountains, and sea) of the place where the measurement of $G$ is done.

A linearity violation of gravitational forces might be predicted by the model. If predicted, it could explain, also, this historical disparity in the measurements of $G$. This work is in progress.

\section{Conclusions}

As a conclusion, this new modeling of space-time retrieves general and restricted relativity. Nevertheless, it is more than a simple Euclidean representation of relativity, as shown by postulate 1 and 2 .

A direct explanation of the Sagnac effect is given by this modeling. Moreover, it is enough to add a third pos- tulate in order to explain those "dark matter mysteries": 1) galaxy speed profiles, 2) speed of the galaxies themselves, inside their group, and deformation of light trajectories in the vicinity of a galaxy, 3) Pioneer anomaly.

In more details, this third postulate conducts a modifycation of Newton's law. This modification is conceived in order to find exactly Newton's law in the specific case of pin pointed masses inside an homogeneous universe, and long distances. Using the model's equation, immediately a correction of Newton's law is noticed in the case of short distances. This first correction occurs in fact for relativistic speed. Now, adjusting this correction in order to fit Newton's law with this new law for some particular distance, yields immediately a theoretical explanation of Pioneer anomaly. This model is also predicting an anomaly for Saturn flyby by Pioneer 11 .

Another result of this correction of Newton's law occurs when the galaxy's stars are introduced in the model. After this, a strong difference appears between the calculated force and Newton's law. The predicted galaxy speed profiles are close to measured speed profiles.

For the mystery of galaxy velocities, the explanation is more direct. This mystery is explained by a different value of $G$, between our case inside the Milky Way, and the case outside any galaxy. Here again, the "third speaker”, which decreases $G$ constant, is the stars of our galaxy.

This model might explain also, after some calculations, the following anomalies:

- Earth flyby anomalies,

- perihelion precession of Saturn,

- disparity of the gravitational constant measurements.

Moreover, and from a theoretical point of view, this study finds a way for space-time determination. At any space-time point, this determination is based upon the matter density distribution throughout the whole universe. This model is compatible by construction with restricted and general relativity. The PPN parameters are exactly the same as for relativity. It seems to be compatible also with gravitation experimental measurements.

As a conclusion, the gravitational model of the three elements theory seems to be validated. As such, this is a validation of the three elements theory itself. This theory is described in [14]. A next step will be to solve the sign issue for the dark matter mystery (speed profiles). The exact comparison with ephemerides must be done also. Another interesting work will be to calculate the linearity violation of gravitational forces predicted by the model, and to compare it to experimental data. It remains also to check if the model described in this article is coherent with other actual physics theories (electromagnetism, quantum mechanics, etc). As an answer to this last question one will notice that this model is in conformity with a 
unifying theory called three elements theory.

\section{REFERENCES}

[1] F. Lassiaille, "A Solution for the Dark Matter Mystery Based on Euclidean Relativity,” 2010. http://lumi.chez-alice.fr/anglais/mystmass.pdf

[2] F. Lassiaille, "Validation of Dark Matter Explanation," 2010. http://lumi.chez-alice.fr/anglais/Calcul_NGC3310.pdf

[3] F. Lassiaille, "Gravitational Model of the Three Elements Theory: Explanation of the Pioneer Anomaly,” 2012. http://lumi.chez-alice.fr/anglais/PioneerExpl.pdf

[4] S. T. Turyshev and V. T. Toth, "The Pioneer Anomaly," Living Reviews in Relativity, Vol. 13, 2010, p. 4.

[5] G. A. Kyazumov, "The Velocity Field of the Galaxy NGC 7541,” Pis'ma v Astronomicheskii Zhurnal, Vol. 6, 1980, pp. 398-401.

[6] G. Galletta, and E. Recillas-Cruz, "The Large Scale Trend of Rotation Curves in the Spiral Galaxies NGC 1068 and NGC 3310,” Astronomy and Astrophysics, Vol. 112, 1982, pp. 361-365.

[7] J. D. Anderson, J. K. Campbell, J. E. Ekelund, J. Ellis, and J. F. Jordan, "Anomalous Orbital-Energy Changes Observed during Spacecraft Flybys of Earth,” Physical Review Letters, Vol. 100, No. 9, 2008, pp. 091102-091105. doi:10.1103/PhysRevLett.100.091102
[8] I. Lorenzo, "The Recently Determined Anomalous Perihelion Precession of Saturn," Astronomical Journal, Vol. 137, No. 3, 2009, p. 3615. doi:10.1088/0004-6256/137/3/3615

[9] G. T. Gillies, “The Newtonian Gravitational Constant: Recent Measurements and Related Studies,” Physical Review Letters, Vol. 60, No. 2, 1997, p. 151. doi:10.1088/0034-4885/60/2/001

[10] M. L. Gershteyn, L. I. Gershteyn, A. Gershteyn and O. V. Karagioz, "Experimental Evidence That the Gravitational Constant Varies with Orientation," Classical Physics, Gravitation \& Cosmology, Vol. 8, No. 3, 2002, pp. 1-6.

[11] M. L. Gershteyn and L. I. Gershteyn, "The Attractive Universe Theory," Australian National University, Redaktsiya Izvestiya Vuzov Radiophysica, 2004. http://hdl.handle.net/1885/41360

[12] W. Michaelis, H. Haars and R. Augustin, "A New Precise Determination of Newton's Gravitational Constant," Metrologia, Vol. 32, No. 4, 1995, pp. 267-270. doi:10.1088/0026-1394/32/4/4

[13] C. H. Bagley and G. G. Luther, "Preliminary Results of a Determination of the Newtonian Constant of Gravitation: A Test of the Kuroda Hypothesis," Physical Review Letters, Vol. 78, 1997, pp. 3047-3050. doi:10.1103/PhysRevLett.78.3047

[14] F. Lassiaille, “Three Elements Theory,” 1999. http://lumi.chez-alice.fr/3elt.pdf 